\title{
Single-particle resonances in a deformed relativistic potential
}

\author{
MENG Jie
}

A variety of structural phenomena in exotic short-lived nuclei far from stability, especially in systems close to the particle drip lines, challenge model descriptions based on the self-consistent mean-field approximation. Because the Fermi level in a drip-line nucleus is very close to the continuum, both weakly-bound states and low-lying positive energy single-particle resonant states are essential to determine the ground state properties of such systems. The research team at Peking University, which has been dedicated to the study of nuclear structure and astrophysics for the last few decades, has examined the evolution of levels close to the continuum threshold and, in particular, the occurrence of single-neutron resonant states as functions of the axial deformation parameter $0 \leqslant \beta \leqslant 0.5$. The study is reported in Issue 53 , No. 4 (April, 2010) of SCIENCE CHINA Physics, Mechanics \& Astronomy.

For an open-shell nucleus close to the drip-line, interesting effects result from the interplay between deformation and low-lying resonances, and therefore the theoretical investigation of single-particle resonant states in deformed potentials is particularly interesting. In the paper by Li et al., the single-neutron states with positive parity have been obtained as solutions of the corresponding single-particle Dirac equation, using the coupled-channels method in coordinate space.

The main conclusion reported by the investigators is that the admixture of $\mathrm{s} 1 / 2$ component determines the occurrence of single-neutron resonant states as functions of the axial deformation parameter.

"In our current work, the main focus is on single-particle resonances in a deformed system, especially those with $\mathrm{m}^{\pi}=1 / 2^{+}$. This is also the foundation for the understanding of a deformed exotic nucleus in Relativistic Mean-Field (RMF) theory and a natural extension of our previous work on spherical cases" noted principal investigator MENG Jie, Professor at Peking University.

It is considered that this research deserves publication because the approach is state-of-art, the work is innovative, the results are of interest, and the paper is well written.

This work was supported by the Major State Basic Research Developing Program, the National Natural Science Foundation in China, and Inter-governmental S\&T Cooperation Project between China and Croatia.

See the article: Li Z P, Zhang Y, Vretenar D, et al. Single-particle resonances in a deformed relativistic potential. Sci China Phys Mach Astron, 2010, 53: 773-778, doi: 10.1007/s11433-010-0161-7 